\title{
Studies on Direct and Indirect Effect of Traits on Seed Yield of Fenugreek (Trigonella foenum-graceum $\mathrm{L}$ )
}

\author{
Sharvan Kumar, V. B. Singh, V. P. Pandey, Manjeet Kumar* and Rohit Bajpai \\ Department of Vegetable Science, Acharya Narendra Deva University of Agriculture \& \\ Technology, Kumarganj, Ayodhya, India \\ *Corresponding author
}

\begin{abstract}
A B S T R A C T
Keywords

Fenugreek,

Trigonella foenumgraceum

Article Info

Accepted:

20 August 2020

Available Online:

10 September 2020

The present investigation was carried out at Main Experiment Station, Department of Vegetable Science, Acharya Narendra Deva University of Agriculture \& Technology, Kumarganj, Ayodhya (U.P.). The path coefficient analysis was carried out from phenotypic and genotypic correlation coefficient to resolve direct and indirect effect of different characters on seed yield per plant. The direct and indirect effect of different characters on seed yield per plant at phenotypic level and genotypic levels was studied. At phenotypic level, harvest index via, test weight (0.574), number of pods per plant $(0.891)$ followed by plant height (0.490), pod length (0.186), and days to maturity $(0.403)$, excreted maximum indirect effect on seed yield per plant. Other character showed negligible value on seed yield per plant. Harvest index and biological yield emerged as a direct contributor to seed yield per plant while harvest index via, days to maturity, plant height, pod length, number of pod per plant and test weight as indirect contributors towards seed yield per plant. Hence due emphasis should be given to these characters during selection for developing high yielding genotypes in fenugreek.
\end{abstract}

\section{Introduction}

Fenugreek (Trigonella foenum-graecum L.) $2 \mathrm{n}=16$, locally known as 'Methi' belongs to the family-Leguminoaceae and sub-familyPapilionaceae, along with it's another cultivated species Trigonella corniculata L. commonly known as Kasuri or Champamethi differing as growth habit, pod seed size and yield potential as serve and multipurpose crop. Fenugreek is grown during Rabi or winter season as a leafy vegetable, seed or leaf spices for human consumption (Som and
Maity 1986, Pandey 1993) fodder for the animal (Jatasra and Lodhi, 1980) and green manure to enrich the soil fertility through nitrogen fixation, i.e. above $283 \mathrm{~kg} \mathrm{~N} / \mathrm{ha}$ (Gill \& Singh 1988). Trigonella comes from Latin words 'little triangle'in reference to triangular shape of small yellowish white flowers. The spices epithet foenum-gracummeans 'Greek hay' and according to Rosengarten, the Romans, who got the plant from Greece where it was a very common crop in ancient times, gave it this name it is also called 'ox horn' or goat horn because the two seeds pods 
projecting in opposite direction usually from the nodes of the stem base that resemble with ox or goat horns. The nutritive value of fenugreek (per 100g of edible portion) as reported by Gupta et al., (1989) viz., moisture $(13.7 \mathrm{~g})$, protein $(26.2 \mathrm{~g})$, carbohydrate $(44.1 \mathrm{~g})$, crude fiber (7.2g), fat (5.8g.), Mineral (3.0g), Calcium (160 mg), Iron(14.1mg), Phosphorus (370 mg), Vitamin A (96 I.U.), Vitamin B (10.34mg), Vitamin $B_{2} \quad(20.2 \mathrm{mg})$, Niacin (1.1mg) and food energy (333cal.).In India, it occupies an area of about 218 thousand hectare with $220 \mathrm{mt}$ annual productions with share of 2.4 percent (Anonymous, 2017-18). Path coefficient provides an effective means of entangling direct and indirect causes of association of selection and measures the relative importance of each causal factor. Hybridization offers high probability for increasing variability for further selection and the greatest possibilities for improvement of fenugreek. The parents for hybridization should be chosen to complete with the breeding objectives and the special attributes of the lines and generally to provide planned genetic variability for subsequent selection. A special technique consisting of diallel or line $\mathrm{x}$ tester mating schemes should be used to determine the combining ability of the varieties that are going to be crossed.

\section{Materials and Methods}

The present investigation entitled "Studies on direct and indirect effect of traits on seed yield of fenugreek (Trigonella foenumgraceum L)" was carried out at Main Experiment Station (Vegetable Research Farm), Narendra Nagar (Kumarganj), Ayodhya (U.P.) India, during Rabi season of 2018-19. Geographically the experimental site falls under humid subtropical climate and is located at $26.47^{\circ} \mathrm{N}$ latitude and $82.12^{\circ}$ Elongitude at an elevation of altitude of 113 meter above the mean sea level. Geographically it falls in north east gangatic alluvial plains of eastern U.P. Ayodhya region. The experimental field had sandy loam soil, low in organic carbon, nitrogen, medium in phosphorous, potash and slightly alkaline $(\mathrm{pH}$ 8.0) in nature. The climate of district Ayodhya is semi-arid with hot summer and cold winter. Maximum rains in this area are received from July to the end of September. The data was recorded at Meteorological Observatory of Acharya Narendra Deva University of Agriculture and Technology, Narendra Nagar (Kumarganj), Ayodhya (U.P.) The experiment was conducted in a Randomized Block Design (R.B.D.) with three replications to assess the performance of $45 \mathrm{~F}_{1}$ hybrids and their 10 parents and one commercial check. The crop was planted in row length spaced $30 \mathrm{~cm}$. apart where, $10 \mathrm{~cm}$. plant to plant spacing was maintained. The eight pure lines of fenugreek were provided by the Acharya Narendra Deva University of Agriculture and Technology, Narendra Nagar (Kumarganj), Ayodhya (U.P.) India and two others commercial varieties collected from IARI, New Delhi and HAU, Hissartaken for this investigation. The observations were recorded on eleven characters viz, Days to $50 \%$ flowering, days to maturity, plant height $(\mathrm{cm})$, number of branches per plant, number of pods per plant, pod length $(\mathrm{cm})$, number of seeds per pod, biological yield per plant $(\mathrm{g})$, harvest-index $(\%)$, test weight $(\mathrm{g})$, seed yield per plant $(\mathrm{g})$. The experimental data was compiled by taking the mean value of the 10 parents and $45 \mathrm{~F}_{1}$ 's and one standard check for all the 11 characters from all the three replications.

\section{Results and Discussion}

The path coefficient analysis was carried out from phenotypic and genotypic correlation coefficient to resolve direct and indirect effect of different characters on seed yield per plant. The direct and indirect effect of different characters on seed yield per plant at 
phenotypic level and genotypic levels. It helps to find out the direct and indirect effect of yield attributes that which is one of great importance to select the superior genotypes. The estimates of correlation coefficients indicate only the inter-relationship of the character but, do not furnish information on the cause and effects relationship. Wright (1921) has devised the analysis of path coefficient to provide effective means of finding out direct and indirect causes of association which permits the critical examination of specific forces acting to produce a given correlation and measure the relative Importance of each casual factor. Dewey and Lu (1959) were first demonstrating the utility of path coefficient analysis in breeding programme using crested wheat grass progenies. Due to the manual association, the development of dependent variables is determined by the degree of direct effect of independent variable and indirect effect excreted via other characters, arising inevitably as an integral part of the growth pattern. Under such complex situations, the total correlation is insufficient to explain the real association for an effective and fruitful manipulation of the characters. The path coefficient analysis was carried out from phenotypic and genotypic path coefficient to resolve direct and indirect effect of different character on seed yield.

The direct and indirect effect of different characters on seed yield at phenotypic level presented in Table 1, 2 and 3. The analysis path coefficient revealed that the highest positive direct effect on seed yield per plant was excreted by harvest index (1.121) flowed by biological yield (0.644) at phenotypic level. At phenotypic level, harvest index (0.837) via, number pods per plant $(0.837)$ followed by test weight (0.540), pod length (0.219), plant height (0.462) and days to maturity (0.360), excreted maximum indirect effect on seed yield per plant. Other character showed negligible value on seed yield per plant. Harvest index, biological yield emergedas a direct contributor to seed yield per plant while harvest index via, days to maturity, plant height, pod length and number of pod per plant as indirect contributors towards seed yield per plant during $\mathrm{E}_{1}$. The analysis path coefficient revealed that the highest positive direct effect on seed yield per plant was excreted by harvest index (1.219) followed by biological yield (0.689) at phenotypic level.

At phenotypic level, harvest index via, test weight (0.588), number pods per plant (0.917) followed by pod length $(0.217)$, plant height (0.498) and days to maturity (0.434), excerted maximum indirect effect on seed yield per plant. Other character showed negligible value on seed yield per plant. Harvest index, biological yield emerged as a direct contributor to seed yield per plant while harvest index via, days to maturity, plant height, pod length, number of pod per plant and test weight as indirect contributors towards seed yield per plant during $\mathrm{E}_{2}$.

The analysis path coefficient revealed that the highest positive direct effect on seed yield per plant was excerted by harvest index (1.188) followed by biological yield (0.679) at phenotypic level. At phenotypic level, harvest index via, test weight (0.574), number of pods per plant $(0.891)$ followed by plant height (0.490), pod length (0.186), and days to maturity (0.403), excreted maximum indirect effect on seed yield per plant. Other character showed negligible value on seed yield per plant. Harvest index and biological yield emerged as a direct contributor to seed yield per plant while harvest index via, days to maturity, plant height, pod length, number of pod per plant and test weight as indirect contributors towards seed yield per plant during over season (pooled) (Table 4-6). 
Table.1 Estimates of direct and indirect effect (phenotypic) of among different characters in fenugreek (E1)

\begin{tabular}{|c|c|c|c|c|c|c|c|c|c|c|c|}
\hline Traits & $\begin{array}{l}\text { Days to } \\
50 \% \\
\text { flowering }\end{array}$ & $\begin{array}{l}\text { Days to } \\
\text { maturity }\end{array}$ & $\begin{array}{l}\text { Plant } \\
\text { height } \\
\text { (cm) }\end{array}$ & $\begin{array}{l}\text { No. of } \\
\text { branches } \\
\text { per plant }\end{array}$ & $\begin{array}{l}\text { Pod } \\
\text { length } \\
\text { (cm) }\end{array}$ & $\begin{array}{l}\text { No. of } \\
\text { pod per } \\
\text { plant }\end{array}$ & $\begin{array}{l}\text { No. of } \\
\text { seed per } \\
\text { pod }\end{array}$ & $\begin{array}{l}\text { Test } \\
\text { wieght } \\
\text { (g) }\end{array}$ & $\begin{array}{l}\text { Biological } \\
\text { yield (g) }\end{array}$ & $\begin{array}{l}\text { Harvest } \\
\text { index }\end{array}$ & $\begin{array}{l}\text { Yield per } \\
\text { plant (g) }\end{array}$ \\
\hline Days to $50 \%$ flowering & -0.048 & 0.010 & 0.000 & 0.000 & 0.009 & -0.007 & 0.000 & 0.020 & -0.091 & 0.111 & 0.004 \\
\hline Days to maturity & -0.028 & 0.018 & 0.000 & 0.000 & 0.008 & 0.026 & 0.007 & 0.013 & -0.053 & 0.360 & $0.352 * *$ \\
\hline Plant height $(\mathrm{cm})$ & -0.001 & 0.001 & -0.001 & 0.002 & 0.008 & 0.027 & 0.004 & 0.008 & -0.174 & 0.462 & $0.337 *$ \\
\hline No. of branches per plant & 0.003 & -0.001 & 0.000 & 0.008 & -0.005 & 0.027 & -0.007 & 0.000 & 0.258 & -0.006 & $0.278 *$ \\
\hline Pod length $(\mathrm{cm})$ & -0.008 & 0.003 & 0.000 & -0.001 & 0.054 & 0.020 & -0.024 & 0.002 & 0.061 & 0.219 & $0.325 *$ \\
\hline No. of pod per plant & 0.004 & 0.005 & 0.000 & 0.002 & 0.012 & 0.092 & -0.001 & 0.005 & -0.033 & 0.837 & 0.921 \\
\hline No. of seed per pod & 0.000 & -0.003 & 0.000 & 0.002 & 0.035 & 0.003 & -0.037 & -0.002 & 0.220 & -0.107 & 0.11 \\
\hline Test wieght (g) & -0.031 & 0.007 & 0.000 & 0.000 & 0.003 & 0.014 & 0.002 & 0.032 & -0.322 & 0.540 & 0.246 \\
\hline Biological yield (g) & 0.007 & -0.001 & 0.000 & 0.003 & 0.005 & -0.005 & -0.013 & -0.016 & 0.644 & -0.695 & -0.07 \\
\hline Harvest index & -0.005 & 0.006 & 0.000 & 0.000 & 0.010 & 0.068 & 0.004 & 0.015 & -0.399 & 1.121 & $0.821 * *$ \\
\hline
\end{tabular}

Residual effect $=0.1034$

*** Significant at $5 \%$ and $1 \%$ probability level respectively

Table.2 Estimates of direct and indirect effect (phenotypic) of among different characters in fenugreek (E2)

\begin{tabular}{|c|c|c|c|c|c|c|c|c|c|c|c|}
\hline Traits & $\begin{array}{l}\text { Days to } \\
\mathbf{5 0 \%} \\
\text { flowering }\end{array}$ & $\begin{array}{l}\text { Days to } \\
\text { maturity }\end{array}$ & $\begin{array}{l}\text { Plant } \\
\text { height } \\
\text { (cm) }\end{array}$ & $\begin{array}{l}\text { No. of } \\
\text { branches } \\
\text { per plant }\end{array}$ & $\begin{array}{l}\text { Pod } \\
\text { length } \\
\text { (cm) }\end{array}$ & $\begin{array}{l}\text { No. of } \\
\text { pod per } \\
\text { plant }\end{array}$ & $\begin{array}{l}\text { No. of } \\
\text { seed per } \\
\text { pod }\end{array}$ & $\begin{array}{l}\text { Test } \\
\text { wieght } \\
\text { (g) }\end{array}$ & $\begin{array}{l}\text { Biological } \\
\text { yield (g) }\end{array}$ & $\begin{array}{l}\text { Harvest } \\
\text { index }\end{array}$ & $\begin{array}{l}\text { Yield per } \\
\text { plant (g) }\end{array}$ \\
\hline Days to $50 \%$ flowering & -0.004 & -0.006 & 0.000 & 0.000 & 0.006 & -0.002 & 0.000 & -0.002 & -0.098 & 0.109 & 0.004 \\
\hline Days to maturity & -0.002 & -0.011 & 0.000 & 0.000 & 0.005 & 0.011 & 0.007 & -0.001 & -0.050 & 0.434 & $0.393 * *$ \\
\hline Plant height (cm) & 0.000 & -0.001 & 0.005 & 0.002 & 0.004 & 0.010 & 0.005 & -0.001 & -0.187 & 0.498 & $0.335^{*}$ \\
\hline No. of branches per plant & 0.000 & 0.000 & 0.001 & 0.009 & -0.005 & 0.010 & -0.009 & 0.000 & 0.278 & -0.007 & $0.278^{*}$ \\
\hline Pod length $(\mathrm{cm})$ & -0.001 & -0.002 & 0.001 & -0.001 & 0.037 & 0.005 & -0.030 & 0.000 & 0.040 & 0.217 & $0.267^{*}$ \\
\hline No. of pod per plant & 0.000 & -0.004 & 0.002 & 0.003 & 0.006 & 0.033 & -0.001 & 0.000 & -0.034 & 0.917 & $0.922 * *$ \\
\hline No. of seed per pod & 0.000 & 0.002 & -0.001 & 0.002 & 0.024 & 0.001 & -0.047 & 0.000 & 0.234 & -0.102 & 0.113 \\
\hline Test wieght (g) & -0.002 & -0.005 & 0.001 & 0.000 & 0.002 & 0.005 & 0.003 & -0.002 & -0.345 & 0.588 & 0.246 \\
\hline Biological yield (g) & 0.001 & 0.001 & -0.001 & 0.004 & 0.002 & -0.002 & -0.016 & 0.001 & 0.689 & -0.749 & -0.07 \\
\hline Harvest index & 0.000 & -0.004 & 0.002 & 0.000 & 0.007 & 0.025 & 0.004 & -0.001 & -0.423 & 1.219 & $0.828 * *$ \\
\hline
\end{tabular}

Residual effect $=0.0718$

*,** Significant at $5 \%$ and $1 \%$ probability level respectively 
Table.3 Estimates of direct and indirect effect (phenotypic) of among different characters in fenugreek (pooled)

\begin{tabular}{|c|c|c|c|c|c|c|c|c|c|c|c|}
\hline Traits & $\begin{array}{l}\text { Days to } \\
\mathbf{5 0 \%} \\
\text { flowering }\end{array}$ & $\begin{array}{l}\text { Days to } \\
\text { maturity }\end{array}$ & $\begin{array}{l}\text { Plant } \\
\text { height } \\
\text { (cm) }\end{array}$ & $\begin{array}{l}\text { No. of } \\
\text { branches } \\
\text { per plant }\end{array}$ & $\begin{array}{l}\text { Pod } \\
\text { length } \\
\text { (cm) }\end{array}$ & $\begin{array}{l}\text { No. of } \\
\text { pod per } \\
\text { plant }\end{array}$ & $\begin{array}{l}\text { No. of } \\
\text { seed per } \\
\text { pod }\end{array}$ & $\begin{array}{l}\text { Test } \\
\text { wieght } \\
\text { (g) }\end{array}$ & $\begin{array}{l}\text { Biological } \\
\text { yield (g) }\end{array}$ & $\begin{array}{l}\text { Harvest } \\
\text { index }\end{array}$ & $\begin{array}{l}\text { Yield per } \\
\text { plant (g) }\end{array}$ \\
\hline Days to $50 \%$ flowering & -0.033 & 0.004 & 0.000 & -0.001 & 0.007 & -0.003 & 0.000 & 0.013 & -0.096 & 0.113 & 0.004 \\
\hline Days to maturity & -0.018 & 0.008 & 0.000 & 0.000 & 0.003 & 0.014 & 0.010 & 0.008 & -0.053 & 0.403 & $0.374 * *$ \\
\hline Plant height (cm) & 0.000 & 0.000 & -0.001 & 0.002 & 0.006 & 0.013 & 0.006 & 0.005 & -0.184 & 0.490 & $0.338^{*}$ \\
\hline No. of branches per plant & 0.002 & 0.000 & 0.000 & 0.012 & -0.005 & 0.013 & -0.011 & 0.000 & 0.274 & -0.007 & $0.281^{*}$ \\
\hline Pod length $(\mathrm{cm})$ & -0.004 & 0.000 & 0.000 & -0.001 & 0.058 & 0.008 & -0.039 & 0.000 & 0.069 & 0.186 & $0.287 *$ \\
\hline No. of pod per plant & 0.002 & 0.002 & 0.000 & 0.003 & 0.010 & 0.046 & -0.002 & 0.003 & -0.034 & 0.891 & $0.921 * *$ \\
\hline Test wieght (g) & -0.021 & 0.003 & 0.000 & 0.000 & -0.001 & 0.007 & 0.004 & 0.020 & -0.340 & 0.574 & 0.247 \\
\hline Biological yield (g) & 0.005 & -0.001 & 0.000 & 0.005 & 0.006 & -0.002 & -0.019 & -0.010 & 0.679 & -0.733 & -0.071 \\
\hline Harvest index & -0.003 & 0.003 & 0.000 & 0.000 & 0.009 & 0.034 & 0.005 & 0.010 & -0.419 & 1.188 & $0.825 * *$ \\
\hline
\end{tabular}

Residual effect $=0.0750$

*** Significant at $5 \%$ and $1 \%$ probability level respectively

Table.4 Estimates of direct and indirect effect (genotypic) of among different characters in fenugreek $\left(\mathrm{E}_{1}\right)$

\begin{tabular}{|c|c|c|c|c|c|c|c|c|c|c|c|c|}
\hline $\begin{array}{c}\text { S.N } \\
\text { o }\end{array}$ & Characters & $\begin{array}{c}\text { Days to } \\
50 \% \\
\text { Flowerin } \\
\text { g }\end{array}$ & $\begin{array}{l}\text { Days to } \\
\text { Maturity }\end{array}$ & $\begin{array}{c}\text { Plant } \\
\text { Height } \\
\text { (cm) }\end{array}$ & $\begin{array}{c}\text { Branches/ } \\
\text { Plant }\end{array}$ & $\begin{array}{l}\text { Pods } \\
\text { Length } \\
\text { (cm) }\end{array}$ & $\begin{array}{l}\text { Pods/ } \\
\text { plant }\end{array}$ & $\begin{array}{l}\text { Seeds/ } \\
\text { Pod }\end{array}$ & $\begin{array}{c}\text { Test } \\
\text { Weight }\end{array}$ & $\begin{array}{l}\text { Biologica } \\
\text { I Yield/ } \\
\text { Plant (g) }\end{array}$ & $\begin{array}{c}\text { Harvest } \\
\text { Index }(\%)\end{array}$ & $\begin{array}{l}\text { Seed } \\
\text { Yield/ } \\
\text { Plant }\end{array}$ \\
\hline 1 & Days to $50 \%$ Flowering & 0.750 & -0.636 & 0.010 & 0.082 & -0.221 & -0.257 & -0.033 & 0.451 & 0.029 & -0.169 & 0.007 \\
\hline 2 & Days to Maturity & 0.874 & -0.546 & 0.024 & 0.248 & -0.384 & 1.680 & -0.497 & 0.334 & 0.032 & -0.971 & $0.794 * *$ \\
\hline 3 & Plant Height $(\mathrm{cm})$ & 0.012 & -0.022 & 0.590 & -0.318 & -0.208 & 0.957 & -0.149 & 0.163 & 0.057 & -0.716 & $0.366^{* *}$ \\
\hline 4 & Branches/ Plant & -0.047 & 0.102 & 0.142 & -1.322 & 0.163 & 1.074 & 0.301 & 0.016 & -0.096 & 0.005 & $0.339^{*}$ \\
\hline 5 & Pods/ Plant & 0.138 & -0.174 & 0.102 & 0.180 & -1.203 & 0.719 & 0.976 & 0.023 & -0.028 & -0.386 & $0.348^{* *}$ \\
\hline 6 & Pods Length $(\mathrm{cm})$ & -0.063 & -0.300 & 0.185 & -0.464 & -0.283 & 3.059 & 0.032 & 0.109 & 0.013 & -1.271 & 1.017 \\
\hline 7 & Seeds/ Pod & -0.021 & 0.230 & -0.075 & -0.338 & -0.997 & 0.083 & 1.177 & -0.012 & -0.085 & 0.171 & 0.133 \\
\hline 8 & Test Weight & 0.926 & -0.498 & 0.263 & -0.059 & -0.076 & 0.908 & -0.038 & 0.366 & 0.205 & -1.501 & $0.495^{* *}$ \\
\hline 9 & Biological Yield/ Plant (g) & -0.119 & 0.094 & -0.183 & -0.693 & -0.182 & -0.221 & 0.549 & -0.409 & -0.183 & 1.250 & -0.097 \\
\hline 10 & Harvest Index (\%) & 0.080 & -0.334 & 0.266 & 0.004 & -0.293 & 2.452 & -0.127 & 0.346 & 0.144 & -1.586 & $0.953 * *$ \\
\hline
\end{tabular}

Residual effect $=0.3543 ; * * *$ Significant at $5 \%$ and $1 \%$ probability level respectively 
Table.5 Estimates of direct and indirect effect (genotypic) of among different characters in fenugreek $\left(\mathrm{E}_{2}\right)$

\begin{tabular}{|c|c|c|c|c|c|c|c|c|c|c|c|c|}
\hline $\begin{array}{c}\text { S.N } \\
\text { o }\end{array}$ & Characters & $\begin{array}{c}\text { Days to } \\
\mathbf{5 0 \%} \\
\text { Flowering }\end{array}$ & $\begin{array}{c}\text { Days to } \\
\text { Maturit } \\
y\end{array}$ & $\begin{array}{l}\text { Plant } \\
\text { Height } \\
\text { (cm) }\end{array}$ & $\begin{array}{c}\text { Branches/ } \\
\text { Plant }\end{array}$ & $\begin{array}{l}\text { Pods } \\
\text { Length } \\
\text { (cm) }\end{array}$ & $\begin{array}{l}\text { Pods/ } \\
\text { plant }\end{array}$ & $\begin{array}{l}\text { Seeds/ } \\
\text { Pod }\end{array}$ & $\begin{array}{c}1000 \\
\text { Grain } \\
\text { Weight }\end{array}$ & $\begin{array}{l}\text { Biologica } \\
\text { I Yield/ } \\
\text { Plant (g) }\end{array}$ & $\begin{array}{c}\text { Harvest } \\
\text { Index }(\%)\end{array}$ & $\begin{array}{l}\text { Seed } \\
\text { Yield/ } \\
\text { Plant }\end{array}$ \\
\hline 1 & Days to $50 \%$ Flowering & 0.188 & -0.267 & 0.000 & 0.008 & 0.021 & -0.102 & 0.000 & 0.162 & -0.018 & 0.016 & 0.009 \\
\hline 2 & Days to Maturity & 0.175 & -0.286 & 0.001 & 0.019 & 0.026 & 0.605 & 0.002 & 0.120 & -0.039 & 0.100 & $0.723 * *$ \\
\hline 3 & Plant Height $(\mathrm{cm})$ & 0.005 & -0.031 & 0.007 & -0.028 & 0.014 & 0.365 & 0.001 & 0.068 & -0.044 & 0.076 & $0.433 * *$ \\
\hline 4 & Branches/ Plant & -0.012 & 0.043 & 0.002 & -0.130 & -0.017 & 0.398 & -0.001 & 0.007 & 0.065 & -0.002 & $0.351 * *$ \\
\hline 5 & Pods/ Plant & 0.033 & -0.063 & 0.001 & 0.018 & 0.120 & 0.203 & -0.005 & 0.011 & 0.005 & 0.036 & $0.359 * *$ \\
\hline 6 & Pods Length (cm) & -0.016 & -0.148 & 0.002 & -0.044 & 0.021 & 1.167 & 0.000 & 0.039 & -0.004 & 0.140 & 1.156 \\
\hline 7 & Seeds/ Pod & -0.005 & 0.098 & -0.001 & -0.031 & 0.098 & 0.038 & -0.006 & -0.005 & 0.074 & -0.013 & 0.247 \\
\hline 8 & 1000 Grain Weight & 0.217 & -0.245 & 0.003 & -0.006 & 0.009 & 0.327 & 0.000 & 0.140 & -0.132 & 0.142 & $0.456 * *$ \\
\hline 9 & Biological Yield/ Plant (g) & -0.036 & 0.123 & -0.003 & -0.092 & 0.006 & -0.054 & -0.005 & -0.204 & 0.091 & -0.216 & $-0.391 * *$ \\
\hline 10 & Harvest Index (\%) & 0.018 & -0.166 & 0.003 & 0.002 & 0.025 & 0.949 & 0.000 & 0.115 & -0.114 & 0.172 & 1 \\
\hline
\end{tabular}
Residual effect $=0.8216$ *** Significant at $5 \%$ and $1 \%$ probability level respectively

Table.6 Estimates of direct and indirect effect (genotypic) of among different characters in fenugreek (Pooled)

\begin{tabular}{|c|c|c|c|c|c|c|c|c|c|c|c|c|}
\hline S.No & Characters & $\begin{array}{c}\text { Days to } \\
50 \% \\
\text { Flowering }\end{array}$ & $\begin{array}{c}\text { Days to } \\
\text { Maturit } \\
y\end{array}$ & $\begin{array}{l}\text { Plant } \\
\text { Height } \\
\text { (cm) }\end{array}$ & $\begin{array}{c}\text { Branches/ } \\
\text { Plant }\end{array}$ & $\begin{array}{l}\text { Pods } \\
\text { Length } \\
\text { (cm) }\end{array}$ & $\begin{array}{l}\text { Pods/ } \\
\text { plant }\end{array}$ & $\begin{array}{l}\text { Seeds/ } \\
\text { Pod }\end{array}$ & $\begin{array}{c}1000 \\
\text { Grain } \\
\text { Weight }\end{array}$ & $\begin{array}{l}\text { Biologica } \\
\text { I Yield/ } \\
\text { Plant (g) }\end{array}$ & $\begin{array}{c}\text { Harvest } \\
\text { Index }(\%)\end{array}$ & $\begin{array}{l}\text { Seed } \\
\text { Yield/ } \\
\text { Plant }\end{array}$ \\
\hline 1 & Days to $50 \%$ Flowering & 0.092 & -0.027 & -0.012 & -0.068 & 0.273 & -0.054 & 0.042 & -0.317 & 0.084 & -0.007 & 0.007 \\
\hline 2 & Days to Maturity & 0.094 & -0.027 & -0.040 & -0.174 & 0.038 & 0.324 & 0.566 & -0.233 & 0.147 & -0.037 & $0.658^{* *}$ \\
\hline 3 & Plant Height $(\mathrm{cm})$ & 0.002 & -0.002 & -0.512 & 0.233 & 0.204 & 0.195 & 0.232 & -0.123 & 0.174 & -0.029 & $0.375^{* *}$ \\
\hline 4 & Branches/ Plant & -0.006 & 0.004 & -0.113 & 1.055 & -0.168 & 0.215 & -0.373 & -0.011 & -0.292 & 0.001 & $0.313^{*}$ \\
\hline 5 & Pods/ Plant & 0.014 & -0.001 & -0.058 & -0.098 & 1.808 & 0.116 & -1.360 & -0.004 & -0.082 & -0.012 & $0.323 *$ \\
\hline 6 & Pods Length (cm) & -0.008 & -0.014 & -0.160 & 0.363 & 0.336 & 0.626 & -0.046 & -0.077 & 0.024 & -0.053 & $0.992 * *$ \\
\hline 7 & Seeds/ Pod & -0.003 & 0.010 & 0.078 & 0.259 & 1.617 & 0.019 & -1.521 & 0.009 & -0.301 & 0.006 & 0.173 \\
\hline 8 & 1000 Grain Weight & 0.109 & -0.023 & -0.235 & 0.045 & 0.030 & 0.180 & 0.050 & -0.268 & 0.601 & -0.057 & $0.432 * *$ \\
\hline 9 & Biological Yield/ Plant (g) & -0.014 & 0.007 & 0.157 & 0.543 & 0.262 & -0.026 & -0.806 & 0.285 & -0.567 & 0.053 & -0.106 \\
\hline 10 & Harvest Index (\%) & 0.009 & -0.015 & -0.228 & -0.010 & 0.336 & 0.499 & 0.139 & -0.233 & 0.457 & -0.066 & $0.887 * *$ \\
\hline
\end{tabular}

Residual effect $=0.2270$

*** Significant at $5 \%$ and $1 \%$ probability level respectively 
Positive direct effect of various traits on seed yield has also been reported by earlier workers, Dashora et al., (2011), Pushpa et al., (2012), Yadav et al., (2013) and Patahk et al., (2014). Finally the path coefficient analysis revealed that focusing plant height, pod length, harvest index and biological yield. Hence due emphasis should be given to these characters during selection for developing high yielding genotypes in fenugreek.

\section{References}

Dashora Abhay; Maloo, S. R.andDashora, L. K.(2011). Variability, correlation and path coefficient analysis in fenugreek (Trigonella foenum-graecum L.) under water limited conditions. Journal of Spices and Aromatic Crops; 20(1):3842.

Dewey, D.R. and Lu, K.H. (1959). "Correlation and path coefficient analysis of components of crested wheat grass seed production. Agron. J., 51: 515-518.

Gill, S.S. and Singh, H. (1988). Effect of planting date and leaf cutting on the seed yield of methi (Trigonella foenumgraecum L.). J. Res. Punjab Agric., Univ., Ludhiana, 25: 206-209.

Gupta, K.K.; Thakral, A. and Wagle, D.S. (1989).Abstract of papers. First National Seminar on seed spices held at
R.A.U. Jaipur, during October 24-25, pp. 511.

Jatasra, D.S. and Lodhi, G.P. (1980). Suitable fodder for grain crop for late sowing. Haryana Fmg., 9(12): 7.

Pandey, S.C. (1993). Improvement of leafy vegetables, In: Advances in Horticulture (K.L. Chadha and G. Kallo, Eds) pp. 325-342. Malhotra Publishing House. New Delhi.

Pushpa, T. N.; Chandregowda, M.; Srikantaprasad, D. and Gowda, A. P. M. (2012). Evaluation of fenugreek

(Trigonella foenum-graecum L.) genotypes for growth and seed yield. Crop Research (Hisar); 43(1/2/3): 238244.

Patahk, A. R.; Patel, A. I.; Joshi, H. K. and Patel, D. A. (2014). Genetic variability, correlation and path coefficient analysis in fenugreek (Trigonella foenumgraecum L.). Trends in Biosciences. 7(4): 234-237.

Som, M.G. and Maity, T.K. (1986). Fenugreek In: Vegetable crops in India (T.K. Bose and M.G. Som, eds.) Naya Prakash. Calcutta, India, pp. 680-686.

Yadav, Yogendra; Yadava, P. S.; Pandey, V. P. and Adesh Kumar (2013). Genetic variability, correlation and path coefficient analysis studies in fenugreek (Trigonella foenum-graecum L.). Asian Journal of Horticulture; 8(2): 456-459.

\section{How to cite this article:}

Sharvan Kumar, V. B. Singh, V. P. Pandey, Manjeet Kumar and Rohit Bajpai. 2020. Studies on Direct and Indirect Effect of Traits on Seed Yield of Fenugreek (Trigonella foenum- graceum L). Int.J.Curr.Microbiol.App.Sci. 9(09): 2942-2948. doi: https://doi.org/10.20546/ijcmas.2020.909.362 